\title{
Regard sociolinguistique sur des formats interactifs en primaire au Sénégal
}

Caroline Juillard

\section{(2) OpenEdition \\ 12 Journals}

Édition électronique

URL : http://journals.openedition.org/esp/3753

DOI : $10.4000 /$ esp.3753

ISSN : 2532-0319

Éditeur

Centre d'Information sur l'Éducation Bilingue et Plurilingue

Édition imprimée

Date de publication : 1 juin 2019

Pagination : 25-36

ISSN : 1127-266X

\section{Référence électronique}

Caroline Juillard, «Regard sociolinguistique sur des formats interactifs en primaire au Sénégal », Éducation et sociétés plurilingues [En ligne], 46 | 2019, mis en ligne le 01 février 2021, consulté le 01 mars 2021. URL : http://journals.openedition.org/esp/3753 ; DOI : https://doi.org/10.4000/esp.3753

Ce document a été généré automatiquement le 1 mars 2021.

(c) CIEBP 


\title{
Regard sociolinguistique sur des formats interactifs en primaire au Sénégal
}

\author{
Caroline Juillard
}

1 Il s'agit dans ce texte d'aborder la question des formats interactifs utilisés, de façon récurrente, dans la relation Maître/élèves du primaire au Sénégal. C'est une relation toujours asymétrique et hiérarchisée, dans lequel le Maître occupe une position haute. C'est pourquoi nous utilisons ici la majuscule $\mathrm{M}$ à l'initiale. On se demandera en quoi la notion d'espace sociolinguistique, relative ici à l'école, à la classe et aux acteurs, peut contribuer à l'appréhension et l'analyse de phénomènes langagiers à la fois répétitifs et changeants. Ce texte propose quelques pistes de réflexion, à partir de recherches effectuées au Sénégal.

2 Nous nous basons sur des observations effectuées entre décembre 2017 et janvier 2018 dans des classes du primaire public sénégalais (en Casamance, à Ziguinchor, dans le quartier Lindiane d'une part, ainsi que dans le village d'Essyl, situé en milieu rural à une quinzaine de kms de la ville, dans le royaume Joola du Bandial, d'autre part). L'une de ces classes pratiquait un enseignement bilingue français/joola, pour des enfants parlant joola, l'une des principales langues de Casamance, dans le quartier et en famille.

3 Nous nous appuyons également sur les résultats des recherches effectuées entre 2001 et 2002 dans la région de Dakar (Dreyfus 2007; Dreyfus et Juillard 2004; Juillard et alii 2005).

\section{Problématique et concepts opératoires}

4 Nous centrons notre approche sur les locuteurs et nous concevons l'école et la classe comme des espaces de socialisation, des lieux de rencontres entre jeunes et adultes, entre adultes, et entre jeunes. Il s'agit de relations langagières quotidiennes, donc fortes, formalisées ou spontanées, qui coconstruisent l'espace sociogéographique vécu et en devenir que sont l'école et la classe, et qui par ailleurs et de façon concomitante 
contribuent à développer les potentialités de l'espace sociolinguistique intériorisé propre à chacun des acteurs.

5 Nous appelons en effet "espace sociolinguistique intériorisé " une construction élaborée par chacun dans la relation aux autres, matérialisée dans et par la variabilité discursive au sein des actes de langage, qui lie les pratiques et leurs significations sociales et/ou personnelles aux situations d'usage, aux lieux, aux personnes, interdépendants au sein d'un espace multidimensionnel vécu (Juillard 2013, 2016; Le Page et Tabouret-Keller 1985; Deprez 2007). Cette élaboration est individuelle autant que collective.

6 L'école, considérée par maints auteurs et sociolinguistes, depuis Fishman (1965), comme un domaine de comportement spécifique, nous intéresse donc au titre des relations sociolinguistiques qui s'y manifestent, mais également au titre du mouvement que le locuteur (l'adulte ou l'enfant) fait quand il passe d'un espace de relations à un autre (par exemple du lieu de vie familial ou du quartier à l'école). Dreyfus (2007) a écrit: "...l'école et la classe représentent des espaces sociolinguistiques en relation avec l'environnement urbain proche et les relations entre le Maître et les élèves au sein des activités dans la classe sont considérées sous l'angle de positionnements interpersonnels opérationnels également dans un espace sociolinguistique plus large ». L'espace sociolinguistique est ici considéré au seul sens de l'orientation socio-spatiale des positionnements et des activités langagières afférentes. Nous nous intéressons également à l'impact de la mobilité des acteurs sur leur répertoire langagier, les pratiques in situ et la diffusion des manières de parler, ceci contribuant à ce que le locuteur construise son espace sociolinguistique de référence propre, tant sur le plan des habitus communicationnels que sur celui des perceptions et des catégorisations. Nous appelons "catégorisation» tant le processus que le produit de l'activité métalinguistique des acteurs qui créent dans leurs pratiques langagières «une dynamique de différenciation continue qui se manifeste en contexte, qui naît de - et dans - cette contextualisation » (Nicolai, 2017, p. 159).

7 Il s'agit donc d'une sociolinguistique orientée sur le locuteur, sa mobilité et la diversité de ses moyens d'expressions. L'investissement physique des locuteurs dans l'espace sociogéographique concerné (école, classe, en l'occurrence) a comme corollaire la fréquence, la répétition et la reproduction des actes de langage qui s'y déroulent et qui le définissent, d'un point de vue sociolinguistique. Ceci peut avoir comme conséquence de considérer l'école et la classe comme des espaces produits par des contraintes sociales fortes. Or, nous voulons y considérer de préférence ce qui est de l'ordre d'un processus historicisé, tant pour les Maîtres que pour les élèves.

8 La relation Maître/élève structure en effet la majorité des actes de langage qui se déroulent dans la classe, tant à l'oral qu'à l'écrit. La répétition de ces actes dans la durée construit le répertoire ad hoc qui s'y déploie et s'acquiert, en lien avec la relation de rôle qui les sous-tend. Des relations de rôle d'un type différent peuvent néanmoins exister et se manifester entre les adultes et les enfants qui se connaissent et se fréquentent dans d'autres lieux (la cour de récréation, le quartier, la ville, etc.)

9 Il en est même pour les relations entre les jeunes. L'école est, après ou parallèlement au quartier, le lieu d'un déploiement des pratiques générationnelles, qui inscrit le jeune dans un univers relationnel où se développent leurs réseaux de sociabilité propres et donc le lieu d'actualisation de ressources langagières qui peuvent avoir été acquises en d'autres lieux. La cour de récréation est l'espace/temps réservé à ce type d'échanges, 
souvent considérés par différents chercheurs en sociolinguistique comme témoignant d'usages novateurs ou " en avance ». La langue des cours de récréation (cf. le créole à Belize, le wolof à Ziguinchor) est signalée par différents sociolinguistes comme celle dont l'usage est, sur le plan de la société au sens large, le plus novateur (cf. Le Page \& Tabouret-Keller, 1985, Dreyfus et Juillard, 2005).

Il y aurait donc coexistence, voire confrontation, de deux ordres de faits de communication qui définissent l'espace "classe» (en tant que lieu et que durée de temps) et qui contribuent à construire ou à valider l'espace sociolinguistique, tant individuel que collectif. Le premier, relatif à la relation Maitre/élève - qui est le plus souvent dans le cadre de la classe une relation de type hiérarchique-, le second relatif à une relation le plus souvent horizontale (mais pas toujours) entre élèves. La classe est conçue comme un espace sociogéographique propre, interdépendant cependant de son environnement (autres classes, cour, école, autres écoles, quartier, ville, pays, etc.), où se déploient des personnes ayant des espaces sociolinguistiques propres et possiblement convergents, du fait, entre autres du partage d'activités communes et de répertoires ad hoc.

11 Nous faisons la proposition suivante: l'analyse du langage utilisé au sein de ces échanges (selon les cas, du français et/ou du wolof ou du joola (langue de Casamance, principalement utilisée dans le quartier), doit prendre en compte l'espace sociolinguistique coconstruit et en devenir au sein duquel, par les actes de parole, se meuvent et se positionnent les acteurs.

\section{Quels sont les observables?}

La proposition retenue ici est de s'intéresser aux phénomènes produits à l'intérieur de l'école et de la classe, en mettant en relief la relation d'interdépendance entre soi et l'environnement, afin de déterminer l'orientation socio-spatiale des locuteurs d'une part (Auzanneau et Trimaille 2017 : 354), la construction et la manifestation des espaces sociolinguistiques, intériorisés et vécus d'autre part (Juillard 2016). Les pratiques langagières, au sein de l'école, comme d'autres pratiques sociales, seraient situées et résulteraient d'un procès de nature sociolinguistique. Quels observables retenir, dans cette optique?

13 On peut à la fois problématiser le lieu, l'école, voire la classe, comme une catégorie socio-spatiale distincte par ce qui s'y déroule et s'y déploie sur le plan langagier, et remettre en question sa clôture, sa "distinction », en considérant que c'est un espace à plusieurs dimensions, en interrelation avec d'autres espaces. S'il semble bien y avoir quelque chose de systématique et répétitif dans la relation entre langue-(s)/élève(s)/ classe ou langue(s)/Maître/classe, les locuteurs, jeunes ou adultes, manifestent néanmoins leur multi-dimensionalité propre (celle de leurs répertoires, pratiques, catégorisations) à l'école. En contexte multilingue, comme l'est la Casamance, et le quartier ou le village où sont situées ces écoles, c'est d'autant plus frappant.

Comment appréhender dans un tel contexte les phénomènes, variables par définition ? Il s'agit, à partir des recherches antérieures, de sélectionner des faits, et des ordres de faits: types de relations, activités, discours, types d'activités de langage et d'actes langagiers, etc., et d'envisager une relation possible avec des éléments saillants de la situation et de l'espace matérialisé. 
15 Ainsi, en ce qui concerne la classe, on peut donc vouloir tout d'abord prendre en considération les faits situés que sont: la présence du tableau noir et le changement de configuration de ce tableau d'un jour à l'autre; le recours à l'ardoise par les élèves : le jeu des ardoises, baissées ou levées à deux mains par les élèves, qui soutient le calcul mental à voix haute (usage du procédé La Martinière ${ }^{1}$ par certains Maîtres); la répartition et l'usage qui est fait des table-bancs, du bureau du Maitre, de l'estrade si elle est présente, et les mouvements des personnes dans ce matériel scolaire, comment les élèves sont assis; ce qui fait partie du décor (armoire, ou cartons entassés, accrochages, mots écrits collés au mur, etc.) et qui peut contribuer à la définition de l'espace. Cet inventaire est motivé par ce qui, dans l'espace, est le support ou soutien d'une activité, d'un type de relations, etc. Nous avons constaté l'importance du tableau noir comme support et organisation de textes à l'étude ou de tâches, et donc, plus que livres ou cahiers même, il est le pivot des relations qui s'établissent entre les différents Maitres et les élèves, dans toutes les classes du primaire que nous avons observées. Le jour et la date y sont toujours indiqués. Une carte du Sénégal peut y être dessinée parfois. Un texte en français, voire en joola (pour la classe bilingue joola/français), des versets coraniques, des opérations de calcul, des consignes de travail, selon le moment, y sont inscrits. Un poème, parfois: «Salut à mon drapeau», par exemple. Le titrage (une exigence de l'Inspection d'Académie) est constant: dans une autre couleur, ou souligné, Activités numériques, par exemple. Ce titrage est d'ailleurs recopié par les élèves dans leurs cahiers. Le tableau sert plus rarement de support où les élèves s'essayent à écrire, l'ardoise est là pour çà et donc ce qui est inscrit sur le tableau, le plus souvent par le Maître, fixe un résultat, une manière de dire, un texte, du vocabulaire et sa graphie correcte, des consignes, les titres des leçons, etc. L'usage du tableau est en général associé à la prise d'une position haute dans l'échange. Parfois les élèves prennent la place du Maître et adoptent alors ce positionnement face à leurs congénères. On peut également mentionner la présence et le rôle de la règle du Maître. Celle-ci, avec laquelle on frappe sur la table ou sur le tableau, sert d'appui illocutoire à l'énoncé du Maître, voire de l'élève qui vient au tableau donner sa réponse à une question posée ou orchestrer les réponses des autres.

D'autres faits situés concourent à la construction de l'espace, en soutenant les activités écrites et orales: les quelques livres et les fiches pédagogiques rédigées par le Maître, en particulier. Dans les fiches, le contenu structurant passe par l'oralisation assortie d'écrit qu'en fait le Maître, du tableau noir aux cahiers. Son utilisation est liée à la formation du Maître ainsi qu'au respect de contraintes académiques (cf. ci-après).

Des éléments extérieurs à la classe (le bureau du directeur, la cour, les toilettes, les autres classes) structurent également l'espace socio-géographique et contribuent au déploiement de relations spécifiques et des activités de langage qui les manifestent.

À observer le déroulé de séances en classe, on peut voir que ces éléments, omniprésents, permettent et soutiennent un certain développement des relations de rôle entre Maître et élèves, voire entre élèves. Si le Maître occupe presque constamment la position hiérarchique haute, dans laquelle il s'exprime de préférence en français (sauf dans la classe bilingue ou lors du cours d'arabe, où ce sont le joola et l'arabe qui sont les vecteurs de cette relation spécifique), et s'il attend des réponses ou des reprises/répétitions en français, tout en se déplaçant dans la classe ou en se tenant à côté du tableau, donnant la parole à tel ou tel, tournant le dos aux élèves pour écrire, il arrive que des relations d'une autre nature s'établissent, en aparté. Au seuil de la 
porte sur la cour, ou près du bureau, lorsque le Maitre demande à un élève d'aller faire une course ou délivrer un message, par exemple, la relation s'établit le plus souvent en wolof.

Parmi les activités de langage, la part dévolue aux diverses leçons est d'autant plus longue que les élèves passent beaucoup de temps à recopier dans leurs cahiers ce que le Maître a lui-même copié au tableau. Ceux que nous avons observé dans l'école de Ziguinchor ont beaucoup de mal à réfléchir sur ces textes ou ces consignes, et à identifier les demandes de l'enseignant. La plupart répètent seulement ce que le Maître oralise, en lien avec ce qui est écrit au tableau. Un grand nombre d'ordres ponctuent la séance : «parle fort ! ", « répète ", « fais une phrase », « taisez-vous », etc. Le chahut ou le conflit entre élèves font partie également des activités/actes de langage; en général, le Maître règle le problème en wolof. Le chant, en mode bilingue, éventuellement, est l'une des activités qui permet de créer une relation en langue entre le Maître et les élèves, avec des répons chantés en chœur. Sa répétition quotidienne fait partie des premiers acquis en langue. D'autres activités de langage moins fréquentes (jeux de l'esprit, où concourent les élèves, conseils de classe) se déploient parfois dans la classe.

Pour la plupart de ces activités, des formats interactionnels fréquents ou "patterns ", relevés dans toutes les classes observées, fondent la relation didactique entre le Maître et l'élève ${ }^{2}$. Selon Dreyfus (2007), il s'agit le plus souvent « d'un format interactionnel spécifique caractérisé par une forme séquentielle organisée autour d'une série de paires adjacentes ${ }^{3} \mathrm{du}$ type questions/réponses ou d'échanges ternaires du type: question-initiation/réponse /évaluation ou feedback) ». Nous marquerons l'intonation montante par le symbole /, et l'intonation descendante par le symbole $\backslash$, situés après le mot ou la syllabe qui porte cette intonation. On a relevé également un format marqué par un schéma intonatif spécifique, montant/descendant, dont l'apparition très fréquente en discours semble aléatoire et ne pas être systématiquement liée à un moment précis de l'échange, ou à une thématique quelconque. Ce format peut être associé à une consigne, à une règle énoncée, et le plus souvent à une paire question/ réponse (partielle ou totale), etc. Voici un exemple du type de celle-ci, relevée en CE2 bilingue (Ziguinchor):

La Maîtresse: on écrit d'abord la con- /

Les élèves, en chœur:-signel

21 Ou bien de celle-ci (en CE2, Essyl):

Le Maître: son infinitif, c'est dor/

Le Maître et les élèves, tous ensemble: c'est dormir|

Ce format interactionnel fera l'objet d'une analyse spécifique ci-après.

D'autres observables sont également nécessaires à l'appréhension de l'espace sociolinguistique déployé par les uns et les autres dans le milieu scolaire. Nous pensons ici à des faits accessibles par observation in situ et entretiens associés, selon les méthodes d'une ethnolinguistique de terrain, tant pour les élèves que pour les enseignants. Il s'agit de connaître leurs répertoires, la temporalité et les environnements de leur acquisition/apprentissage/formation, la dimension variable de leurs pratiques langagières, ainsi que leurs représentations/perceptions/ catégorisations de ce qui se passe en langue et en situation, dans divers espaces socio- 
géographiques situés, dont l'espace scolaire. Il suffirait sans doute de se pencher sur quelques personnes choisies à l'aide de critères de différenciation explicites et discutés.

\section{Proposition d'analyse}

d'eux :

Il s'agit de tenter ici une analyse de certains échanges recueillis, à la lumière de la problématique posée, et de se demander en quoi le recours à la notion d'espace(s) sociolinguistique(s) éclaire et enrichit cette analyse.

"C'est dans et par l'interaction que l'on devient membre d'une société, d'un groupe ou d'une communauté de pratiques" (Mondada 2000: 114) (nous soulignons). Les phénomènes retenus devraient donc nous permettre d'appréhender l'espace différemment, comme un procès et non seulement comme un produit. Qu'en est-il donc $\mathrm{du}$ format interactif que nous avons retenu, du fait de sa fréquence? Il ne semble pas que cette fréquence soit liée à des facteurs identifiables dans le hic et nunc. Il semblerait qu'on ne puisse en analyser l'apparition et l'usage que dans l'historicité de ce qui se passe en milieu scolaire, depuis longtemps. Il y aurait donc une répétition/reprise par tous, ensemble, à l'instigation du Maître. On ne peut l'analyser et le comprendre qu'en centrant l'approche sur le Maître, en position haute dans une telle interaction.

En voici de nouveaux exemples :

a) Le Maître ici écrit le titre de la leçon au tableau et se tourne vers les élèves, tout en écrivant, comme pour les associer à ce qu'il est en train de faire :

$$
\begin{aligned}
& \text { M J'ai mis IV Conju- / } \\
& \text { E - gaison \} }
\end{aligned}
$$

b) Le Maître utilise le format de bout en bout pour montrer aux élèves ce qu'il attend $M$ il y a zéro/ bonbon\, donc on va mettre/ zéro réponsel

c) La réponse est toujours induite par la forme de la question posée :

$M$ «avant $»$, c'est le /

E passé $\backslash$

30 M reprend: le passé (et il écrit le mot au tableau).

Ou encore :

M ce soir, après le manger, à 17h, vous allez vous / repo-/

E-ser $\backslash$

32 Contraintes formelles sur la réponse, contrainte de la répétition du format et de la relation $\mathrm{M} / \mathrm{E}$ associée, contrainte de la forme écrite de la langue malgré l'oralisation du format, contraintes sociales associées. Ce ne sont que des contraintes, et ce format répété ne génère aucune liberté pour la prise de parole individuelle. Il est intéressant de mettre ces observations en relation avec ce que les auteurs déjà cités, Bray, Clarke et Stephens (1986: 87 et suivantes) disent de l'individualisme dans la culture et l'éducation islamiques.

On a constaté, d'une manière générale comme dans les exemples ci-dessus, que le Maître dirige toutes les prises de parole. Il est le chef d'orchestre d'un ensemble de personnes qui interagissent avec lui, comme d'une même voix. Sur un mode rythmique 
scandé, le plus souvent, les élèves reprennent en l'imitant ce qu'a dit le Maître, ou donnent la réponse attendue, parce qu'initiée déjà dans le discours du Maître et soutenue par le schéma intonatif. Le jeu des routines interactionnelles (reprises collectives, répétitions de formats stéréotypés), qui ramène toujours à la forme écrite de la langue, a comme effets majeurs de réduire la gamme des ressources communicatives et de construire une "surnorme scolaire» (cf. Dreyfus 2005, 2007), laquelle s'installe dans un habitus lié, tant à la langue qu'à l'espace socio-géographique situé. C'est cela qui construit l'espace vécu, intériorisé par chacun, et par tous, en l'occurrence.

De même que la répétition des opérations de calcul cadrées par le PLM, la répétition de cette routine interactionnelle contribue à souder la relation Maître/élèves, sinon la transmission d'un savoir nouveau. La répétition de cette routine associée à l'usage du français renforce la position haute du Maître et donc la portée symbolique et la fonction haute de la langue concernée (français, ou arabe, lors de l'apprentissage par cœur des versets du Coran).

L'usage de ce format est toujours le fait du Maître, jamais des élèves (sauf lorsque certains prennent la posture du Maître). Il ne se produit qu'en classe. C'est un usage aléatoire, au hasard, indépendant des contenus. L'intérêt ou le but de ce format interactionnel semble être de re-saisir, de re-mobiliser les élèves, souvent occupés à recopier ce qui est écrit au tableau. Il peut également servir à introduire une nouvelle séquence, à vérifier un acquis, sinon une réelle compréhension. Sa fréquence semble liée assez nettement au degré d'insécurité du Maître dans sa fonction. Cependant, tous les Maîtres, même les plus expérimentés, l'utilisent à un moment ou un autre. Il y a donc là un savoir partagé, hérité, et son usage contribue à créer une culture scolaire, incorporée dans cet acte de langage stéréotypé.

On peut se demander pourquoi ces Maîtres utilisent si peu de questions ouvertes et donnent la réponse dans la question posée. Si l'on replace cet usage spécifique dans l'historicité de ce qui se passe, on doit se poser cette question: d'où vient ce format?

Y a-t-il là une reprise, par des Maîtres peu ou mal formés, malgré leur implication et leur dévouement, de manières de dire et de faire héritées de l'école coloniale ? C'est ce qui nous a été suggéré par une ancienne institutrice de l'école française au Sénégal, qui s'indignait devant les données que nous lui avons montrées, un «massacre pédagogique » d'après elle.

38 Peut-on trouver d'autres espaces de transmission, où ce format serait également fréquent? On peut penser à l'école coranique: ce format interactif a été également observé dans les classes d'arabe dispensées par des oustases ${ }^{4}$, au sein de l'école publique.

Des éléments de comparaison manquent pour donner à ce format sa pleine mesure culturelle, linguistique et sociale. On ne peut que faire l'hypothèse de sa pérennité, de sa reprise, dans le contexte des formations discursives en classe. Il faudrait réaliser des entretiens approfondis avec les Maîtres, les Inspecteurs d'Académie et les formateurs d'enseignants pour, d'une part mesurer leur prise de conscience du phénomène, d'autre part envisager avec eux la question de sa transmission, par qui, où et quand. On en vient donc à la grande question de l'historicité de la culture scolaire, au Sénégal et dans les anciennes colonies françaises. 
cas est d'autant plus intéressant, à notre avis, qu'il exemplifie la dimension sociale et historicisée de la construction par réitération de formats interactifs, d'un espace sociolinguistique identifiant la relation Maître/élève en classe.

\section{En manière de conclusion}

41 La reproduction sociale de formats interactifs spécifiques et ritualisés, utilisés dans l'apprentissage scolaire et les rituels communicationnels en classe, n'est pas dissociable des langues en contact qui les véhiculent (français, arabe, joola). Elle doit être considérée pour ce qu'elle est, dans des environnements situés, d'un point de vue sociolinguistique, et être mise en relation, si cela est possible, avec des formats de même type, utilisés dans d'autres circonstances, d'autres environnements. Il s'agit de montrer tant l'interdépendance des milieux ou des espaces socio-géographiques liés d'une façon ou d'une autre aux apprentissages, que de mettre en évidence le fait que ce qui se construit, en langue comme en représentations, dans l'espace sociolinguistique de chacun est fortement contingent de ces répétitions, reprises, scansions, dans lesquelles la langue en position "haute" s'incorpore, qu'il s'agisse de l'arabe, du français ou du joola. Nous mettons sur un pied d'égalité symbolique ces trois langues, lorsqu'elles sont utilisées par le Maître à des fins d'apprentissage, comme dans la classe bilingue que nous avons observée. Il ne nous est pas apparu cependant que le format en question ait été aussi utilisé lorsque la classe est conduite en joola. Cela reste néanmoins à vérifier.

Existe-t-il au sein de la communauté familiale des transmissions-apprentissages langagiers, également façonnés dans des formats interactifs spécifiques (histoires racontées?), qui contribueraient à co-construire dans l'interaction l'espace sociolinguistique de chacun ou du groupe? Si l'on fait l'hypothèse de la «reproduction», au sens bourdieusien du terme, alors on a là, dans ce format exemplaire, un aspect significatif de l'histoire vécue de la langue, les espaces sociolinguistiques s'interpénétrant et se reproduisant, d'une catégorie de la population, les Maîtres, de différents âges, à différentes époques, à leurs élèves, qui vont à leur tour devenir des Maîtres, sans la possibilité d'un retour critique sur cet usage, qui semble devenir consubstantiel de la fonction.

Nicolaï souligne :

«Ce n'est pas dans un espace abstrait que la 'langue' prend racine mais dans la matérialité du contact entre les individus, les populations, à travers les effets d'historicité que nous produisons dans l'actualisation de nos existences » (Nicolaï 2007: 3).

L'espace sociolinguistique est donc vécu, il est multidimensionnel et fondé, incorporé, dans les actes de langage. La population réunie en classe illustre ce fait. L'empirie de l'observation et de la description permet seule d'accéder à cet espace, de voir comment il fonctionne et se construit ou se reproduit pour chacun. 


\section{BIBLIOGRAPHIE}

AUZANNEAU M. \& TRIMAILLE C. 2017. L'odyssée de l'espace en sociolinguistique , Langage et société n ${ }^{\circ}$ 160-161: 349-367.

Bray M., Clarke P. B. \& STePhens D. 1986. Education and Society in Africa, Londres, Edward Arnold Publishers.

DEPREZ C. 2007. Langues et espaces vécus dans la migration: quelques réflexions, Langage et société $\mathrm{n}^{\circ}$ 121-122: 247-257.

DREYFuS M. 2005. Pratiques de classes, interactions et appropriation du français en milieu multilingue, Appropriation du français et construction de connaissances via la scolarisation en situation diglossique, Actes du colloque international de l'Université Paris X Nanterre, février 2005, Ouvrage multimédia sur CD rom sous la direction de C. NOYAU.

DREYFUS M. 2007. Point de vue à propos de l'analyse d'interactions en milieu multiculturel et plurilingue, pp. 179-198 in M. AUZANNEAU (dir.), La mise en cuvre des langues dans l'interaction, Paris, L'Harmattan.

DREYFUS M. \& JUILLARD C. 2004. Enseignement non formel dans la banlieue de Dakar: un espace scolaire alternatif, entre activités novatrices et pratiques ritualisées » dans Penser la francophonie, concepts, actions et outils linguistiques. Paris AUF: 355-376.

DREYFUS M. \& JUILLARD C. 2005. Le plurilinguisme au Sénégal. Langues et identités en devenir, Karthala. FISHMAN J. 1965. Who speaks what language to whom and when?, La linguistique 2: 67-88.

JUILLARD C. avec la collaboration de DREYFus M., MoRSLY D., NAPON A., ThIAM Nd. 2005. Dynamiques sociolinguistiques (scolaires et extrascolaires) de l'apprentissage et de l'usage du français dans un cadre bi- ou plurilingue (langues de migrants, langues locales) sur les axes ouest-africain et franco-africain, ARP du Réseau sociolinguistique et dynamique des langues, AUF, C. JUILLARD (dir. scientifique), Rapport consultable sur le site http://www.sdl.auf.org., 103 p.

JUILLARD C. 2016, L'espace sociolinguistique et les actes de langage, La linguistique 52: 91-124.

JUILLARD C. 2013. La description de l'empirie du langage et la question de l'espace sociolinguistique, pp. 173-186 in C. DE FÉRAL (ed.), In and Out of Africa. Languages in Question, In Honour of Robert Nicolaï, Vol. 1, Language Contact and Epistemological Issues, BICLLL 130.

Le PAge R. \& TABouret-Keller A. 1985. Acts of Identity: Creole-Based Approaches to Language and Identity, Cambridge, CUP.

MONDADA L. 2000. Apports de l'ethnométhodologie et de l'analyse conversationnelle à la description de l'acquisition dans l'interaction, pp. 112-116 in MARQUILLO LARRUY, M. (ed.), Questions d'épistémologie en didactique du français, Université de Poitiers,.

Nicolai R. 2007. Le contact des langues: point aveugle du linguistique, Journal of Language Contact, Thema 1, www.jlc-journal.org

NicolaI R. 2017. Signifier, Essai sur la mise en signification. Parcours dans l'espace épistémique et dans l'espace communicationnel ordinaire, ENS Editions. 


\section{NOTES}

1. Le Procédé La Martinière ou PLM, pour le calcul mental, consiste à susciter des réponses par écrit, sur l'ardoise, au signal donné par le Maître. Ceci a pour effet de discipliner l'interrogation et d'obliger toute la classe à participer activement.

2. Selon Bray, Clarke \& Stephens (1986), «discovery methods are not widely used in Africa. One factor is the relatively low skill and self-confidence of the teachers, for it is easier to use chalk and talk methods than to guide discovery learning» (p.138). Ce constat reste actuel. De plus, «many teachers and parents favour an organized environment, and distrust what they consider to be the potential chaos of children learning in their own ways and at their own speeds, often with considerable noise». Ces remarques s'appliquent tout à fait aux classes que nous avons observées en Casamance. Lorsque le Maître est expérimenté et a confiance en lui, la relation M/E tend à être davantage centrée sur l'élève, même si le Maître garde toujours une position hiérarchique dominante.

3. Paire adjacente: deux énoncés contigus produits par deux locuteurs différents, la production du premier énoncé (membre) de la paire exerce une contrainte très forte sur le tour suivant, selon le principe de dépendance conditionnelle.

4. Un oustase est un maître de l'école coranique. Il y enseigne le Coran et l'arabe. L'enseignement de l'arabe a été introduit dans les classe primaires publiques. Ces classes sont actuellement tenues par des oustases et par des enseignants d'arabe formés par l'Inspection académique.

\section{RÉSUMÉS}

In che cosa può contribuire la nozione di spazio, da un punto di vista sociolinguistico, all'acquisizione e all'analisi di fenomeni linguistici ripetitivi e allo stesso tempo mutevoli, come quelli che si manifestano all'interno del contesto scolastico? Questo testo propone qualche traccia di riflessione.

How can, how does, the notion of space contribute, from a sociolinguistic point of view to understanding and analyzing language phenomena that are all at once repetitive and changing, such as those that occur in a school environment? This text offers a few lines of thought to follow up on the subject.

\section{INDEX}

Keywords : Senegal, Casamance, French, Joola/Diola, sociolinguistic space, interaction format, primary school/elementary school

Parole chiave : Senegal, Casamance, francese, joola, spazio sociolinguistico, formato interattivo, scuola primaria 
AUTEUR

CAROLINE JUILLARD

Université René Descartes, Paris (France) 